\title{
Enzymatic Synthesis of Nucleoside Triphosphates. \\ Does It Involve An Ion-Radical Path?
}

\author{
Anatoly L. Buchachenko ${ }^{1}$, Maxim E. Grigoryev ${ }^{2}$, Natalya N. Breslavskaya ${ }^{3}$, Dmitry A. \\ Kuznetsov ${ }^{1,2}$
}

1 -N. N. Semenov Institute for Chemical Physics, Russian Academy of Sciences, Moscow 119991, Russia

2 - N. I. Pirogov Russian National Research Medical University, Russian Federal Ministry of Health, Moscow 117997, Russia

3 - N. S .Kurnakov Institute for General and Inorganic Chemistry, Russian Academy of Sciences, Moscow119991, Russian Federation

Corresponding Author: Professor D. A. Kuznetsov, kuznano@mail.ru

\begin{abstract}
Accumulation and release of energy in the nucleoside triphosphate enzymatic synthesis and hydrolysis does not limited to a routine energy consuming nucleophilic mechanism. These processes require an overcoming the large energy barrier exceeding a total value of accumulated or released energy level by at least $3-4$ times $(\sim 10 \mathrm{kcal} / \mathrm{mol})$. This energy is supposed to be taken from the mechanical compression of the catalytic site and used to form $\mathrm{P}-\mathrm{O}$ chemical bond by a direct nucleophilic addition of phosphate to nucleoside diphosphate (ADP as an example). A new, energetically "cheap", ion-radical mechanism of the ATP biosynthesis has been proposed due to the observation of magnetic isotope and magnetic field effects on the ATP synthesis. This mechanism is about to generate a compression energy to "spend" on a partial dehydratation of magnesium ion inside the nucleotidyl transferase catalyric site (energy cost of this process is 3-5 $\mathrm{kcal} / \mathrm{mol}$, i.e. by 2-3 times less than a total accumulated or released energy). Dehydration of this ion is to increase its electron affinity and hence to stimulate an electron transfer from $\mathrm{ADP}^{3-}$ to $\mathrm{Mg}^{2+}$. This reaction is a starting point of the ionradical mechanism considering the molecular mechanics of enzymatic machines and its quantum chemistry background as well. To the contrast of a hardly controllable nucleophilic path, the ion-radical mechanism might be turned on/off by a targeted delivery of paramagnetic magnesium ions, ${ }^{25} \mathrm{Mg}^{2+}$, towards the phosphate transferring enzyme catalytic site. The magnesium isotope substitution is easily reachable by the endo-osmotic pressure techniques, which makes it attractive for further biotechnological and/or pharmacological application(s).
\end{abstract}

Indexing terms / Key words: Mass-independent isotope effect; magnetic isotope effect; ion-radical pairs; singlet triplet spin conversion; Coulomb hyperfine coupling; enzymatic phosphorylation; magnesium isotopes.

\section{Academic Discipline and Sub-Disciplines}

Chemistry / Biochemistry.

\section{Subject Classification}

Chemical physics and biophysics / chemical enzymology.

TYPE (METHOD / APPROACH)

Mini Review.

\section{Council for Innovative Research}

Peer Review Research Publishing System

\section{Journal: Journal of Advances in Chemistry}

Vol. 4, No. 3

editor@cirworld.com

www.cirworld.com, member.cirworld.com 


\section{INTRODUCTION / BACKGROUND}

In terms of the broadly adopted conventional paradigm, the ATP synthesis is a nucleophilic reaction. It proceeds as the attack of the attaching phosphate residue towards a terminal P-atom in ADP to form a terminal P-O bond accumulating in ATP energy $(\sim 10 \mathrm{kcal} / \mathrm{mol})$. The reaction is known to be catalyzed by $\mathrm{Mg}^{2+}$ ion. The latter is considered to coordinate reactants in the catalytic site keeping them on the reaction trajectory to facilitate nucleophilic attack and probably slightly modify their reactivity due to a partial redistribution of charges in the reactants. According to this paradigm, the nucleophilic attack requires unification of the two closed electronic shells of the reacting molecules. Such pressing of one of these shells into another one needs to overcome a huge energy barrier caused by the repulsive potential of the exchange forces. The latter is may lead to an essential increase of the short distances needed for the reaction to get launched, so the Coulomb hyperfine coupling potential would not capable to compensate this short distance repulsive potential $(1-5)$.

This undisputable physical argument has been already proven by numerous quantum chemical calculations of phosphorylation energy barriers engaging the molecular dynamics techniques as well $(4,5,7)$. The nucleophilic phosphoryl group transfer requires $42-46 \mathrm{kcal} / \mathrm{mol}$ (1); nucleophilic attack of hydroxyl to hydrolyze ATP needs to overcome a $39 \mathrm{kcal} / \mathrm{mol}$ energy barrier (2). That means that the accumulation as well as the release of a relatively small amount of energy $(\sim 10 \mathrm{kcal} / \mathrm{mol})$ requires high energy cost which is by 3-4 times higher than the accumulated or released energies themselves.

A source of energy needed to cover the energy expenses for energy-consuming ATP synthesis is a compression of the catalytic site which is accompanied by conversion of the protein mechanical energy into the chemical energy of $\mathrm{P}-\mathrm{O}$ bond in ATP. The energy pumping of the catalytic site is due to a trans-membrane electric potential (like in the case of ATP-synthase) or owing to thermal fluctuations in kinases; the latter were called by Oster as the Darwin's Brownian motors (3).

\section{A new, energetically "low-cost", mechanism of the ATP synthesis}

It turns out that the purified kinase directed ATP synthesis as well as the isolated mitochondria ATP production depends on which type of magnesium isotope is in catalytic site. The rates of ATP synthesis driven by enzymes in which $\mathrm{Mg}^{2+}$ ions were presented predominantly by magnetic nuclei ${ }^{25} \mathrm{Mg}$ were found to be $2-3$-fold higher compared to the rates shown by the same enzymes under the same conditions but possessing the non-magnetic, spinless nuclei of ${ }^{24} \mathrm{Mg}$ and ${ }^{26} \mathrm{Mg}$ (4-7). There was no difference in the ATP-producing activities of enzymes with ${ }^{24} \mathrm{Mg}^{2+}$ or ${ }^{26} \mathrm{Mg}^{2+}$ ions.

A discovery of such a huge mass-independent, nuclear-magnetic isotope effect is itself a reliable evidence to support the fact that the ATP synthesis is a radical or ion-radical process. Paramagnetic intermediates - ion-radicals and ionradical pairs - are to participate in this reaction. A mere fact that the ATP synthesis rate depends on the magnesium nuclei spin shows the key role of magnesium ion in a conversion of protein domain compression energy into the energy of $\mathrm{P}-\mathrm{O}$ chemical bond. It is indeed the ion-radical (quantum mechanical not thermodynamic) mechanism lying a background for a new paradigm of enzymatic nucleoside triphosphate synthesis.

This new mechanism works in both oxidative and substrate phosphorylation paths (Figure 1).

Figure 1. An ion-radical path in the ATP-synthase functioning (Ref.: 4, 7).

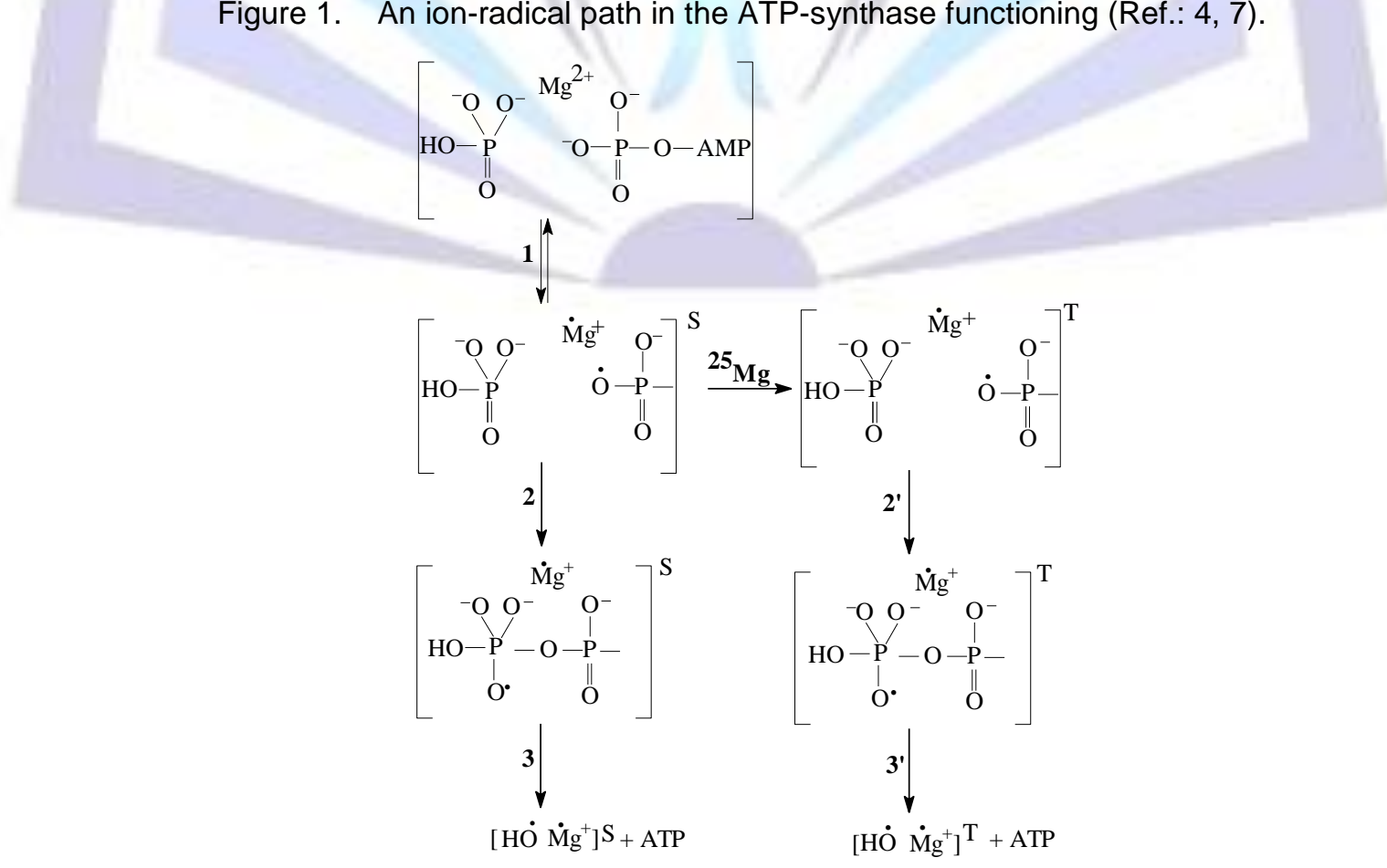


As a first step, Figure 1 implies an electron transfer from the terminal phosphate group of $\mathrm{ADP}$ to $\mathrm{Mg}^{2+}$ ion; it generates the primary ion-radical pair, composed of the radical-cation $\mathrm{Mg}^{+}$and oxy-radical of ADP (reaction 1). This is a key reaction where molecular dynamics and chemistry works together, a point where mechanics of the ATP-synthesizing molecular machines meets the reactants conversion mode. Due to the total spin conservation primary ion-radical pair, as to any other of the thermally generated radical pair, this temporarily remains in a singlet spin state. The next step of the mechanism proposed is the phosphorylation itself in which the ADP oxy-radical attacks the $\mathrm{P}=\mathrm{O}$ chemical bond of inorganic phosphate (reaction 2). The resulting oxy-radical decomposes via $\beta$-scission of the $\mathrm{P}-\mathrm{O}$ chemical bond (reaction 3). This leads to the ATP formation along with a final ion-radical pair $\left(\mathrm{HO} \mathrm{Mg}^{+}\right)$; the latter regenerates $\mathrm{Mg}^{2+}$ in the reaction of back electron transfer:

$$
\left(\mathrm{HO} \dot{\mathrm{Mg}}^{+}\right) \stackrel{\mathrm{H}^{+}}{\longrightarrow} \mathrm{H}_{2} \mathrm{O}+\mathrm{Mg}^{2+}
$$

The singlet channel related phosphorylation rates (reactions 1-3, Figure 1) are to get suppressed by the spin allowed reverse electron transfer mode in the primary ion-radical pair which, in turns, regenerates the starting reactants and hence decreases the further ATP yield.

However, once the ${ }^{25} \mathrm{Mg}^{2+}$ promoted hyperfine coupling is the case, its unpaired electron stimulate the singlet-triplet spin conversion of the primary ion-radical pair and transforms it into a triplet pair where the returned electron transfer is spin - forbidden $(7,8)$. This "newborn" triplet channel of phosphorylation (reactions 2' and 3') provides an additional yield of ATP which increases the total production of ATP by 2-3 times. The final ion-radical pair in the triplet channel undergoes fast triplet-singlet conversion due to electron spin relaxation (in $\mathrm{OH}$ the spin relaxation time is about $10^{-11} \mathrm{~s}$ ) and again regenerates $\mathrm{Mg}^{2+}$ ion in the reaction (I) $(8-10)$.

The essence of the magnetic isotope effect in ATP synthesis, therefore, is all about a magnetic interaction of the unpaired electron in ${ }^{25} \mathrm{Mg}^{+}$radical with a magnetic nucleus followed by the singlet-triplet spin conversion of the ion-radical pair formed. This creates an additional, "fast-running" reaction channel in the ATP synthesis. It would be safe to define that as a remarkable way for the nuclear spin control over the chemical reactivity.

In terms of the routine nucleophilic paradigm, this "parallel" ion-radical mechanism looks revolutionary which is, nonetheless, has a firm enough experimental background beyond. Thus, it has been proven by the magnetic isotope effects of calcium and zinc $\left({ }^{43} \mathrm{Ca}^{2+},{ }^{67} \mathrm{Zn}^{2+}\right.$ in several reactions programmed by purified nucleotidyl kinases and protein kinases $(7-10)$

A separate evidence to support this ion-radical mechanism is a discovery of the magnetic field impact on the ATP synthesis - Figure 2 (10).
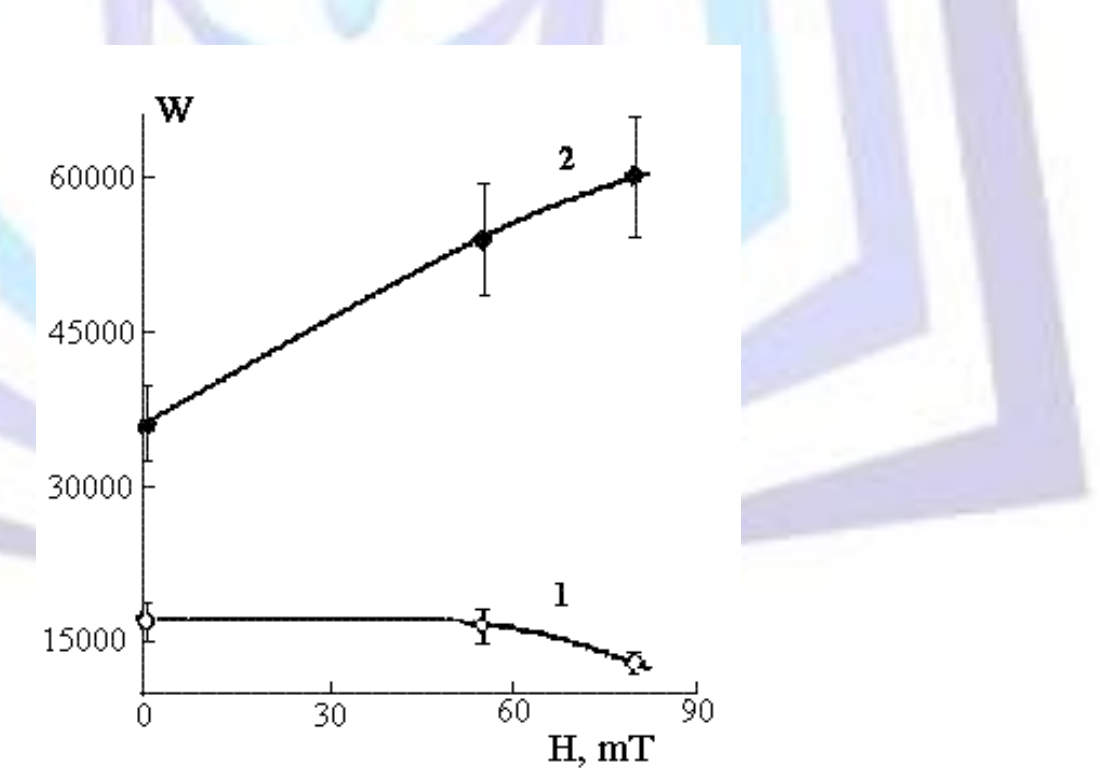

Figure 2. The rate $\mathrm{w}$ of the ATP synthesis by creatine kinase with ${ }^{24} \mathrm{Mg}^{2+}(1)$ and ${ }^{25} \mathrm{Mg}^{2+}(2)$ ions as a function of magnetic field (Ref.: 10).

Noteworthy, the in vivo processed magnetic isotope effect hyper-stimulation of the ATP synthesis was detected in several living organisms (rats, rabbits, goats) - Figure 3. The yield of ATP in the heart muscle tissue was found to be 3-6fold higher when ${ }^{25} \mathrm{Mg}^{2+}$ ions were delivered - by specific cationite nanopharmacophore (PMC16) - compared to ${ }^{24} \mathrm{Mg}^{2+}$ ions $(11,12)$. 


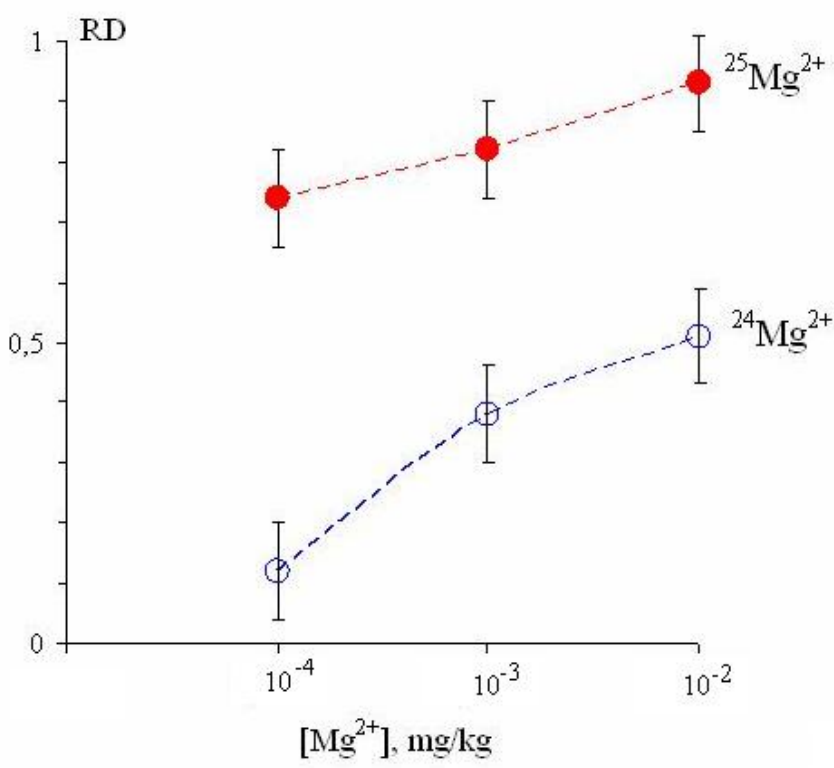

Figure 3. The recovery degree (RD) of ATP production in rats as a function of amount of the heart muscle targeted magnesium ions. RD stands for the extent of restoration of a hypoxia-suppressed myocardium tissue ATP content, i.e. zero RD means a total ATP deplete, while $R D=1.0$ shows a complete restoration of normal pre-hypoxia myocardium ATP level (Ref.: 13).

Recently, the ATP production directed by two species of creatine kinase in a presence of magnesium isotopes was reexamined (14). Neither isotope effect nor magnetic field effect were found in contrast to the above specified results. However, in both samples of $\mathrm{MgCl}_{2}$ employed in this particular study (14), a high level of $\mathrm{Fe}^{2+}$ contaminations were presented (14.6 and $9.7 \mu \mathrm{g} / \mathrm{mL}$ respectively). This corresponds to the iron contents of about 3 and $2 \%$, respectively. Such a high concentration of magnetic $\mathrm{Fe}^{3+}$ (or $\mathrm{Fe}^{2+}$ ) ions does not kill ion-radical mechanism of the ATP synthesis but destroys its nuclear spin selectivity and deletes the magnetic isotope/magnetic field effects since the singlet-triplet spin conversion is controlled by spin relaxation here rather than by the hyperfine coupling or by Zeeman interaction $(7,13)$.

To reveal the protocol mistake allowed to happen in study (14), a special tests were conducted using a set of iron concentrations taken as 200-1000-fold lower than in experiments described in ref. 14 (15). The results presented in (14) are perfect but conclusions are erroneous (15).

\section{How does ATP-synthesizing machine function?}

Figure 1 is nothing but a scheme to illustrate the idea of how the ion-radical mechanism looks like and why the magnetic isotope effect appears. But for at least two reasons, it is hardly possible to apply this scheme to ATP synthesis in its broad biological meaning.

First, ADP is an electron donor situated right in the enzyme catalytic site being coupled with a hydrated $\mathrm{Mg}^{2+}$ ion $(7$, 16). For deductive simplicity, this is not shown in Figure 1.

Secondly, while accepting an electron from ADP, this magnesium ion stay surrounded by few known amino acid residues and, predominantly, by water molecules $(13,16)$.

Electron transfer (reaction 1, Figure 1) does not occur in water where the metal ions are highly hydrated; its first coordination sphere consists of six water molecules. However, a remarkable property of enzymes is this: in the reactive state, when the enzyme domains are drawn together to unite substrate and ADP, they squeeze water molecules out of the catalytic site (16) and partly dehydrate $\mathrm{Mg}\left(\mathrm{H}_{2} \mathrm{O}\right)_{n}{ }^{2+}$ ion. The following removal of the water molecules increases both positive charge $q(\mathrm{Mg})$ on the magnesium and its electron affinity, i.e. the energy of electron attachment $E_{a}$ calculated at the B3LYP/6-31G* level of DFT theory (17-19) (Table1). lonization potential of ADP ${ }^{3-}$ was then estimated equal to 4.1eV, as the energy of electron detachment from magnesium pyrophosphate complexes modeling $\left[\mathrm{ADP}^{3-}\right] \cdot \mathrm{Mg}\left(\mathrm{H}_{2} \mathrm{O}\right)_{m} ;$ thus was shown to be almost independent on $m$ (19). Taking $E_{a}$ from Table 1, the one may calculate a total energy $E$ as a difference between $E_{a}$ and $E_{d .}$. It is shown in Figure 3 as a function of $n$, the number of water molecules in coordination sphere of magnesium ion. It is in fact the energy of reaction

$\mathrm{Mg}\left(\mathrm{H}_{2} \mathrm{O}\right)_{n}{ }^{2+}+\left[\mathrm{ADP}^{3-}\right] \cdot \mathrm{Mg}\left(\mathrm{H}_{2} \mathrm{O}\right)_{m} \rightarrow \mathrm{Mg}\left(\mathrm{H}_{2} \mathrm{O}\right)_{n}{ }^{+}+\left[\mathrm{ADP}^{2-}\right] \cdot \mathrm{Mg}\left(\mathrm{H}_{2} \mathrm{O}\right)_{m}$

When the value of $n$ is falling down and water molecules are gradually removed, the electron affinity of $\mathrm{Mg}\left(\mathrm{H}_{2} \mathrm{O}\right)_{n}{ }^{2+}$ ion increases. At $n=12$, the energy becomes positive so the reaction becomes exothermic and ATP synthesis is allowed. These arguments provide answers to the questions: What really the molecular machines are needed for? Why do they (and only they) have an exclusive possibility to produce ATP? 
Table1. The charges $q(\mathrm{Mg})$ and energy of electron detachment $E_{a}$ for $\mathrm{Mg}\left(\mathrm{H}_{2} \mathrm{O}\right)_{n}{ }^{2+}$ ions (Ref.19).

\begin{tabular}{ccc}
\hline$N$ & $q(\mathrm{Mg})$ & $E_{a}, \mathrm{eV}$ \\
\hline 0 & 2.00 & 15.4 \\
1 & 1.73 & 13.2 \\
2 & 1.51 & 11.4 \\
3 & 1.31 & 9.5 \\
4 & 1.17 & 7.8 \\
5 & 1.09 & 7.3 \\
6 & 1.01 & 6.5 \\
7 & 0.99 & 6.2 \\
8 & 0.95 & 6.0 \\
10 & 0.88 & 5.2 \\
12 & 0.84 & 4.8
\end{tabular}

Natably, a "dry magnesium" is to provide the right conditions needed to induce the singlet - triplet spin conversion in ion-radical pair by making possible a direct connection between both the donor and the recepient of electron $(7,13)$. The electron transfer energy is found to be then closely related to a magnesium hydratation degree $(13$, 19) - Figure 4.

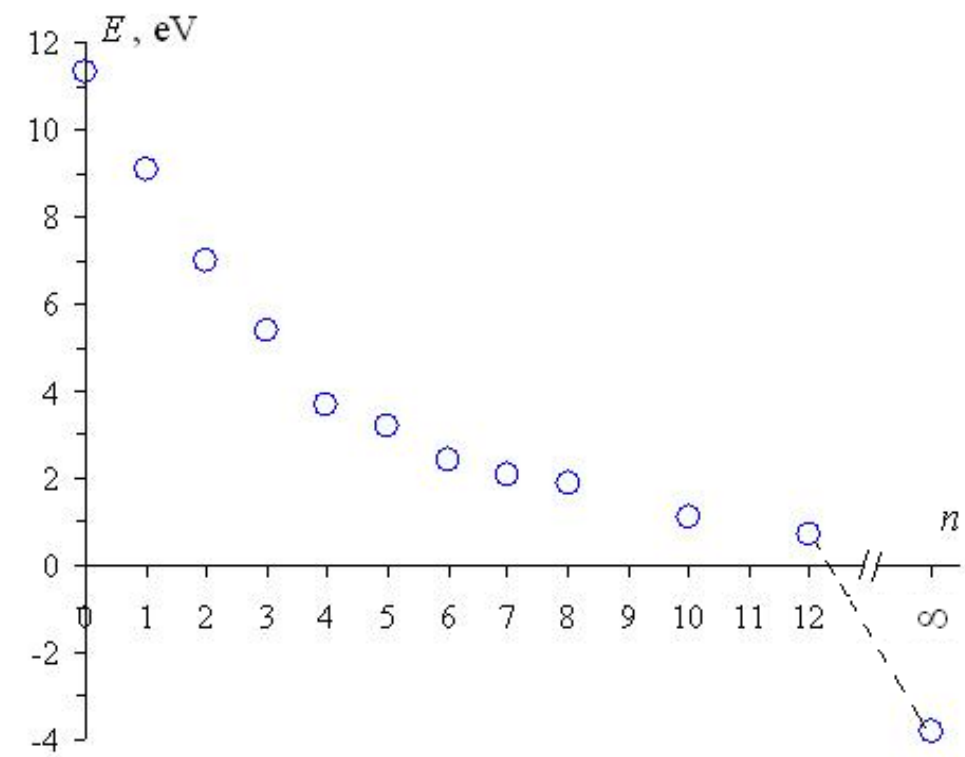

Figure 4. Energies of the electron transfer as a function of $n$, the number of water molecules in the $\mathrm{Mg}\left(\mathrm{H}_{2} \mathrm{O}\right)_{n}{ }^{2+}$ ion (Ref.: 13, 19). 
Following this mechanism, i.e. through a partial dehydration of magnesium ion, the energy of enzyme macromolecule compression is to be spent on the removal of water out of the ion hydrate shell which activates this ion as an electron acceptor. There is no need to destroy the first, tightly bound, coordination shell with $n=6$. In order to make allowed electron transfer as well as the ATP synthesis, it is just enough to remove weakly bound water out of an external hydrate shell with $n \geq 12$. In this process, the total energy "expenses" takes about 2-3 times lower energy "to spend" compared with a total accumulated amount of energy itself - as seen from Figure $4(13,19)$.

Thus, physical activation of magnesium ion reached by its partial dehydration and following electron transfer are low energy consuming processes. Both of them are the steps in a mechanism of conversion of the enzyme catalytic site compression energy into the energy of terminal P-O bond in ATP. Dependence of the ATP synthesis on the nuclear magnetic moment of magnesium and on magnetic field is a specific signature of the ion-radical mechanism. It is a reliable test to distinguish two major routs for ATP synthesis, nucleophilic and ion-radical ones.

\section{ATP Synthesis: nucleophilic versus ion-radical mechanism}

An ion-radical mechanism of enzymatic ATP synthesis has three crucial advantages.

First, unlike uncontrollable nucleophilic mechanism, it can be controlled by modulation of magnesium ions concentration, by isotope substitution and external magnetic field (10).

Second, being perfectly controllable, the ion-radical channel can be artificially switched on by delivering of $\mathrm{MgCl}_{2}$ (or, even better, of ${ }^{25} \mathrm{MgCl}_{2}$ ) into a hypoxia-suffering heart muscle tissue to stimulate ATP synthesis in vivo and prevent or correct numerous metabolic heart muscle disorders of many sorts $(11,12)$ related to the deficiency of ATP. For these purposes a special magnesium ion carrier based on some new porphyrin adduct of cyclohexyl-fullerene-C 60 (PMC16 type nano-cationite) has been designed (20). The ability to stimulate ATP synthesis in the heart muscle of the living organisms is demonstrated by in vivo experiments. Injection of doxorubicin was to promote a significant damage to the local myocardial ATP synthesis making it suppressed by about $70 \%$. Then the PCM16 loaded with ${ }^{24} \mathrm{MgCl}_{2}$ or ${ }^{25} \mathrm{MgCl}_{2}$ was injected and after that a recovery of the ATP production up to initial, pre-doxorubicin, level was observed. The extent of recovery as a function of magnesium concentration is shown in Figure 3. Evidently, there is a large isotope effect: $\left[{ }^{25} \mathrm{Mg}\right] \mathrm{PMC}$ stimulates ATP synthesis by $2-3$ times more efficiently than $\left[{ }^{24} \mathrm{Mg}\right] \mathrm{PMC}$. This is a first observation of the isotope effect manifesting itself in the living organism and hence showing the potential of this specific effect to be applied as an efficient remedy for heart diseases treatment. Noteworthy, a pharmacological potential of ${ }^{25} \mathrm{Mg}^{2+}$ ions looks even more attractive because high level of ATP is reached without an increase of the hazardous superoxide radical yield.

The third "benefit" of the ion-radical mechanism is its low energy cost. Indeed, this mechanism does not require to overcome huge energy barrier of the nucleophilic attack. Even a low level of the compression is sufficient to remove a weakly bound water molecules out of the magnesium ion hydrate shell and, hence, to induce an electron transfer as a starting, key reaction of the ATP synthesis. A low energy cost is exactly the way to provide a high efficiency of ATP synthesis along the ion-radical path.

A discovery of the ion-radical mechanism does not exclude a nucleophilic path in enzymatic ATP synthesis. However, it creates a new enigma: why these routes, energy cheap and energy expensive, highly efficient and low efficient, coexist?

No doubt, the source of energy needed to overcome high energy barrier in a nucleophilic attack is a compression of the catalytic site. In order to attach the phosphate group to ADP this compression must be quite significant. But on the way of a hard compression of reactants the ion-radical mechanism lies since it is supposed to be switched on even at rather weak compression. In other words, an ion-radical mechanism should always precede the nucleophilic one. We need to answer the question, under what conditions one or another mechanism dominates. The answer is expected to come from the inspection of the ATP synthesis as a function of the magnesium ion concentration.

There are two states of $\mathrm{Mg}^{2+}$ ions inside the catalytic site: in one of them the ion is tightly bound with ADP pyrophosphate residue, in another one metal ion is weakly bound being coordinated by amino acid residues and water molecules. Namely this ion is supposed to be responsible for the ion-radical mechanism since it accepts electron donated by ADP.

It is clear that the $\mathrm{Mg}^{2+}$ ions are to get tightly bound to the ADP pyrophosphate residues at low magnesium concentrations. In this case, the weakly bound hydrated ions are absent. Under these conditions, a nucleophilic mechanism operates while the ion-radical one is switched off. At the high magnesium concentrations, to the contrary, an excess of catalytic ions turns the ion-radical mechanism on.

This prediction is in a perfect agreement with experimental observations. It has been shown that at the low magnesium concentrations, the resulting isotope effect is either small (creatine kinase) or totally absent (pyruvate kinase, glycerophosphate kinase). This leads to a conclusion that the low ion concentrations (close to physiological values) makes the nucleophilic mechanism to dominate. To the opposite, the high magnesium concentrations exceeding the normal physiological values are to turn the ion-radical path on. This property is also exhibited in experiments with $\mathrm{Ca}^{2+}$ and $\mathrm{Zn}^{2+}$ ions $(8,9)$.

The intracellular concentration of metal ions is rather low (15), so that the dominating source of ATP in living organisms is thought to be a nucleophilic reaction. The ion-radical mechanism functions under conditions when the concentration of metal ions is rather high. However, due to local concentrations induced by fluctuations of the distribution or inhomogeneous adsorption of metal ions in cells and mitochondria the contribution of the ion-radical mechanism of ATP 
synthesis in living organisms is not negligible. It was tested by in vivo experiments (Figure 3 ) and demonstrates that both mechanisms, nucleophilic and ion-radical, coexist and function independently.

\section{CONCLUSION}

Being a quantum mechanical, ion - radical process, ${ }^{25} \mathrm{Mg}^{2+}$ - promoted magnetic isotope effect manifests itself in a broad variety of enzymatic reactions as long as they relates to a $\mathrm{Mg}^{2+}$ - dependent phosphate transfer (kinases supefamily, ATPsynthase, nucleotidyl polymerases). The Coulomb hyperfine coupling induced singlet - triplet conversion of ${ }^{25} \mathrm{Mg}^{2+}-$ formed ion-radical pairs generated inside the enzyme catalytic site stands beyond this phenomenon. This might operate over the enzymatic phosphorylation metabolic paths in a way of their nuclear - magnetic control. A marked efficiency of this spin selectivity driven regulation of enzyme functioning provides some reasons for optimism regarding the further perspective of biotechnological and/or pharmacological applications of magnetic isotopes of the enzyme - belonging bivalent metal ions.

\section{REFERENCES}

1. Cavalli, A., De Viro, M., Recanatini, M. (2003) Density functional study of the enzymatic reaction catalyzed by cyclindependent kinase. Chem. Commun., 44, 1308 - 1309

2. Akola, J., Jones, R.O. (2003) ATP hydrolysis in water - a density functional study. Amer. J. Phys. Chem. B, 107, 11774 $-11783$

3. Oster, G. (2002) Darwin's motors. Nature, 456, 248 - 254

4. Buchachenko, A.L., Kouznetsov, D.A., Arkhangelsky, S.E., Orlova, M.A., Markaryan, A. (2005) Magnetic isotope effect of magnesium in the phosphoglycerate kinase phosphorylation. Proc. Nat. Acad. Sci. U.S.A., 102, 10793 - 10796.

5. Buchachenko, A.L., Kouznetsov, D.A., Breslavskaya, N.N, Orlova, M.A., (2008) Magnesium isotope effect in enzymatic phosphorylation. Amer. J. Phys. Chem. B, 112, 2548 - 2556

6. Buchachenko, A.L., Kouznetsov, D.A., Arkhangelsky, S.E., Orlova, M.A., Markaryan, A. (2005) Spin biochemistry: intramitochondrial phosphorylation is a magnesium nuclear spin controlled process. Mitochondrion, 5, 67 - 70.

7. Buchachenko, A.L. (2009) Magnetic Isotope Effect in Chemistry and Biochemistry, Nova Science Publishers: New York.

8. Buchachenko, A.L., Kouznetsov, D.A., Breslavskaya, N.N., Shchegoleva, L.N., Arkhangelsky, S.E. (2011) Calcium Induced ATP Synthesis: Isotope Effect, Magnetic Parameters and Mechanism. Chem. Phys. Lett., 505, 130 - 135

9. Buchachenko, A.L., Chekhonin, V.P., Orlov, A.P., Kouznetsov, D.A. (2010) Zinc-related magnetic isotope effect in enzymatic ATP synthesis. A medicinal potential. Intern. J. Molec. Med. Adv. Sci., 6, 34-37

10. Buchachenko, A.L...Kouznetsov. D.A. (2008) Magnetic field affects enzymatic ATP synthesis. J. Amer. Chem. Soc. 130, 12868-12869

11. Rezayat, S., Boushehri, S., Salmanian, B., Omidvari, A., Tarighat, S., Esmaeli, S., Sarkar, S., Amirshahi, N., Alyautdin, R., Orlova, M., Trushkov, I., Buchachenko, A., Kuznetsov, D. (2009) The porphyrin-fullerene nanoparticules to promote overproduction of ATP in myocardium. European J. Medicinal Chemistry, 44, $1554-1569$

12. Amirshahi, N., Alyautdin, R., Sarkar, S., Rezayat, S., Orlova, M., Trushkov, I., Buchachenko, A., Kuznetsov, D. (2008) Fullerene-based low toxic nanocationite particules to treat hypoxia. Archives of Medical Research, 39, 549 - 560

13. Buchachenko, A.L., Kouznetsov, D.A., Breslavskaya, N.N. (2012) Chemistry of Enzymatic ATP Synthesis: An Insight through the Isotope Window. Chemical Reviews, 112: 2042 - 2058

14. D.Grotty, G.Silkstone, S.Poddar, R.Ranson, A.Prina-Mello, M.Wilson, J.M.D.Coey. (2012) Reexamination of magnetic isotope and field effects on adenosine triphosphate production by creatine kinase Proc. Natl. Acad. Sci. USA DOI:10.1073/pnas.1117840108.

15. Arkhangelsky S.E., Karpov G.G., Glavin G.G., Kuznetsov D.A., Buchachenko A.L. (2013) Isotopic catalysis and isotopic analysis. Russ. J. Phys. Chem. B., 7, 8-11.

16. Lahiri, S.D., Wang, P.F., Babbit, P.C., McLeish, M.J., Kenyon, G.L., Allen, K.N. (2001) The 2.1 A structure of Torpedo californica creatine kinase. Biochemistry, 41, 13861- 13867

17. Frisch, M.J. (2003) Gaussian 03, Gaussian Inc., Pittsburgh, PA

18. Neese F. (2001) Density Functional and Semiempirical program package, Version 2.1, revision 72, 2001, Max Planck Institut für Strahlenchemie, Mülheim

19. Buchachenko, A.L., Kouznetsov, D.A., Breslavskaya, N.N. (2010) lon-radical mechanism of enzymatic ATP synthesis: DFT calculation and txperimental control. Amer. J. Phys. Chem. B, 114, $2287-2292$

20. Amirshahi, N., Alyautdin, R., Sarkar, S., Rezayat, S., Orlova, M., Trushkov, I., Buchachenko, A.L., Kuznetsov, D. (2008) New porphyrin adduct for medicinal use. Intern. J. Nanoscience, 8, 113-135. 